\title{
The influence of membrane bound proteins on phase separation and coarsening in cell membranes
}

\author{
Thomas Witkowski, ${ }^{a}$ Rainer Backofen ${ }^{a}$ and Axel Voigt*ab \\ Received 20th April 2012, Accepted 29th June 2012 \\ DOI: $10.1039 / \mathrm{c} 2 \mathrm{cp} 41274 \mathrm{~h}$
}

A theoretical explanation of the existence of lipid rafts in cell membranes remains a topic of lively debate. Large, micrometer sized rafts are readily observed in artificial membranes and can be explained using thermodynamic models for phase separation and coarsening. In live cells such domains are not observed and various models are proposed to describe why the systems do not coarsen. We review these attempts critically and show within a phase field approach that membrane bound proteins have the potential to explain the different behaviour observed in vitro and in vivo. Large scale simulations are performed to compute scaling laws and size distribution functions under the influence of membrane bound proteins and to observe a significant slow down of the domain coarsening at longer times and a breakdown of the self-similarity of the size-distribution function.

\section{Introduction}

\subsection{Motivation}

Cell membranes play a crucial role, not only by defining the boundary of a cell, but also by taking an active part in cell functioning. Cell membranes consist of a lipid bilayer composed of various types of lipids, polysaccharides and proteins, all strongly interacting with each other. These interactions induce a lateral heterogeneity, which is believed to be crucial for the functionality of the membrane. ${ }^{1}$ The introduced concept of lipid rafts is today understood as heterogeneity as a result of interactions of lipids with proteins and the cytoskeleton, as well as effects due to curvature. Lipid rafts have been implicated in a number of important cellular processes including signal transduction, membrane trafficking and protein sorting, see ref. 2 for a review. Cell membranes can be understood as a two-phase system consisting of the lipid raft phase, enriched in cholesterol, proteins and saturated lipids, which is dispersed in a matrix phase. In model membranes, the two phases are typically associated with a lipid-ordered and a lipid-disordered phase, for which phase separation can be observed. The behaviour of these two systems observed in vitro and in vivo is different. While for cell membranes a heterogeneity on a submicrometer scale persists, model membranes show phase separation and coarsening. ${ }^{3}$ Why do raft domains develop on a submicrometer

${ }^{a}$ Department of Mathematics, TU Dresden, Dresden, Germany.E-mail: thomas.witkowski@tu-dresden.de, rainer.backofen@tu-dresden.de, axel.voigt@tu-dresden.de;

Fax: + 49-351-46337096; Tel: + 49-351-46334266

${ }^{b}$ Center of Advanced Modeling and Simulation, TU Dresden,

Dresden, Germany. Fax: + 49-351-46337096;

Tel: $+49-351-46334187$ scale instead of coarsening until reaching a macroscopic length scale as it happens in model membranes? We summarize possible explanation for this discrepancy between the experimental results in vitro and in vivo, especially large-scale phase separation vs. dynamically stable microdomains and give an explanation using large scale phase-field simulations.

\subsection{Theoretical models for microdomain formation}

The simplest explanation is to understand lipid rafts as transient compositional fluctuations driven by thermal noise. ${ }^{4}$ This requires that the temperature of the in vivo system is above the critical temperature at which phase separation occurs. The domain size is then related to the static correlation length in the system, which was reported in ref. 4 to be $20 \mathrm{~nm}$ at $37^{\circ} \mathrm{C}$, which is in good agreement with typical domain sizes for rafts. However, the lifetime within this approach is much smaller than experimental estimates for raft lifetimes. Further experimental verification is thus needed to verify the speculation of equilibrium fluctuations as the origin of the stabilization of microdomains.

Another possible origin for the formation of microdomains is the presence of membrane proteins. ${ }^{5}$ The proteins are thereby immobile and have a favorable energetic interaction with the domain boundary. Thus phase separation and coarsening may occur in pure immiscible lipid systems, but it does not occur in vivo due to the presence of membrane proteins. In this scenario the domain size is determined by a balance between line tension and the interaction strength between the protein and the domain boundary as well as the number of proteins. Effectively this has the same effect as a line-active component reducing the line tension. An estimate given in ref. 6 proposes the protein density to be rather high to sustain stable raft domains. Our investigation 
will be similar to this hypothesis. A further consequence of the stabilization of small domains was pointed out in ref. 7. Due to logarithmically slow domain growth, the implication is that the domains themselves would be very long-lived and such domains may perhaps be too stable for biological purposes.

A similar reasoning is given in ref. 8 by postulating hybrid lipids as line-active components which reduce the line tension. Hybrid lipids are thereby lipids with a fully saturated hydrocarbon chain and a partially unsaturated chain. This decreases the coarsening process and for strong interaction strengths it might also lead to trapping of small domains, such that microdomains can become thermodynamically stable. An experimental verification for this scenario is not yet known.

We can also view the two lipid monolayers that constitute a membrane as two coupled binary fluids, each with its own dynamical properties. If phase coarsening in both layers proceeds with different speed, e.g. as a result of different friction with the environment, and the fluids in both layers are energetically coupled, then the phase separation dynamics will differ from that of an independent fluid layer. Indeed, this leads to a temporary arrest of the phase separation in one layer and a violation of the dynamic scaling hypothesis ${ }^{9}$ in such situations. The arrest reflects the pinning of large domains by the small ones in the apposed fluid. Also this explanation is rather speculative and requires further experimental verification.

Pinning can also result from interactions with the membrane skeleton. In ref. 10 it is shown that phase separation in twocomponent lipid membranes can be strongly affected by such interactions. Domains dynamically change their shape, but stay pinned to the filaments of the skeleton and eventually prevent large-scale phase separation. The domain size is largely determined by the characteristic compartment size of the filament network. The model predicts that coarsening is strongly slowed down at intermediate stages and crosses over to an extremely slow logarithmic coarsening. Thus depending on the pinning density and the characteristic compartment size one can expect that the domain growth stops, when domains reach the characteristic size. Such behaviour was observed in ref. 10-12. We will also consider this scenario and concentrate on pinning, either as a result of membrane bound proteins or due to interactions with the cytoskeleton. Due to the presence of randomly ordered pinning sites, the introduced interaction is similar to that of the random-field Ising model. ${ }^{13}$

Further possible scenarios to explain the raft hypothesis, which include protein-lipid interactions, membrane curvature effects and lipid recycling, are summarized in ref. 6. It should also be noted that the coarsening process is influenced by the bulk fluid surrounding the membrane. This has been considered using different numerical approaches e.g. in ref. 14-16.

\subsection{Membrane proteins and raft domains within a phase-field description}

A current review on mesoscale domain organization in plasma membranes, ${ }^{17}$ which is based on single-molecule imaging in living cell membranes, indicates that probably all of the described phenomena are coupled to each other in a hierarchical way. The membrane was shown to consist of compartments of $40-300 \mathrm{~nm}$ in diameter due to the partitioning of the plasma membrane by the skeleton and membrane proteins which anchor to the skeleton, raft domains of size 2-20 nm which basically stay within the compartment, as well as complexes of membrane associated proteins which are $3-10 \mathrm{~nm}$ in size. These three hierarchically organized mesostructures are proposed to explain the fundamental functional organization of the membrane. To describe this behaviour in a theoretical model we will concentrate on the interplay of pinning sites resulting from membrane proteins and the membrane skeleton and the dynamics of raft domains following the theoretical investigations of ref. 5,10,11 and 18. While all these models are based on Monte Carlo simulations we will consider a mesoscopic continuum model based on a phase-field approximation, which has the advantage of computational efficiency and allows to be solved on realistic membrane shapes to incorporate curvature effects. Furthermore it can be easily coupled to the external field in the extracellular matrix and the cytoplasm to study their influence on the dynamics of raft domains.

Phase-field models have already been used to study phase separation in lipid membranes. Phase-field simulations on spherical domains ${ }^{19}$ clearly indicate the same scaling behaviour as on planar domains and allow to reproduce the observed separation and coarsening phenomena in giant vesicles, ${ }^{3}$ see Fig. 1. The observed coarsening phenomenon is purely driven by line tension. An influence of the curvature of the geometry on the scaling behaviour is therefore not observed. This is in contrast to other pattern formation studies on surfaces, where the geometry imposes restrictions on the shape of the pattern, see e.g. ref. 20-23.

In ref. 24 and 25 the effect of curvature is investigated and an interplay of the line tension and bending, and its influence on domain growth, budding and fission, is studied, which allows to reproduce the observed bulging of domains in ref. 26. The effect of flow is investigated in ref. 9 and 15 for planar domains and a breakdown of dynamic scaling due to secondary nucleation and pinning is demonstrated, respectively. Recently this is also considered in ref. 27 on curved domains. Various mathematical investigations provide the theoretical basis for the phase-field approach to model phase separation in lipid membranes. ${ }^{28,29} \mathrm{We}$ here extend this theory to consider pinning sites, see also ref. 7,30 and 31. As we are concerned only on the coarsening behaviour under the influence of pinnings we restrict

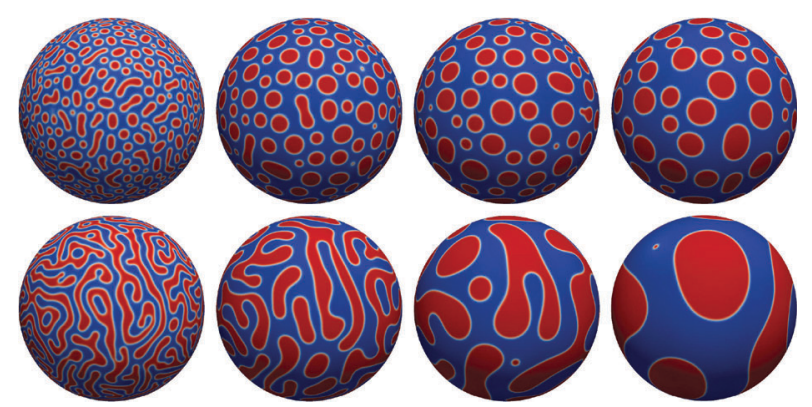

Fig. 1 Domain ripening and spinodal decomposition of a binary lipid system on a spherical domain. Time evolution for a phase distribution of $40: 60$ (top row) and $50: 50$ (bottom row) at various times. A randomly perturbed distribution is used as initial condition. The simulations are performed using parametric finite elements on an unstructured triangular mesh approximating a sphere. 


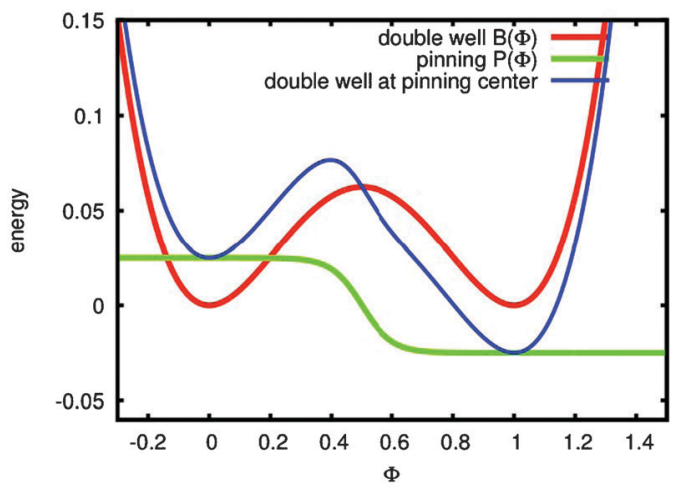

Fig. 2 Locally modified double well potential to model pinning sites. The modification keeps the minima at the original positions.

ourselves to planar domains and neglect all other effects. The underlying model uses the free energy

$$
F_{\mathrm{PF}}[\phi]=\int \frac{1}{2} \varepsilon \Gamma^{2}|\nabla \phi|^{2}+\frac{1}{4 \varepsilon} B(\phi)+V(x) P(\phi) \mathrm{d} \Omega
$$

with phase-field variable $\phi$, interface parameter $\varepsilon$, surface tension parameter $\Gamma$, double well potential $B(\phi)=\phi^{2}(1-\phi)^{2}$ and localisation potential $V(x)=\sum_{i}^{N} \exp \left(-\frac{\left(x-x_{i}\right)^{2}}{\sigma^{2}}\right)$ with the pinning positions $x_{i}$ and the width of the pinning sites $\sigma$. At the pinning centers the energy of phase $\phi=1$ is reduced compared to phase $\phi=0$. This is modeled by $P(\phi)=$ $\tanh (a(0.5-\phi))$. Thus, locally an asymmetric double well potential is introduced. The asymmetric potential is still minimized by $\phi=0$ and $\phi=1$ if the parameter $a$ is big enough, see Fig. 2. We use $(\varepsilon, \Gamma, \sigma, a)=(3.33,0.003,0.055,4)$. This leads to an interface width $w=\sqrt{ } 2 \Gamma \varepsilon=0.085$.

We consider a conserved evolution which leads to a modified Cahn-Hilliard model

$$
\frac{\partial \phi}{\partial t}=-\Delta \frac{\delta F_{\mathrm{PF}}[\phi]}{\delta \phi}=-\Delta \mu_{\mathrm{PF}}
$$

with chemical potential

$$
\mu_{\mathrm{PF}}=-\varepsilon \Gamma^{2} \Delta \phi+\frac{1}{4 \varepsilon} B^{\prime}(\phi)+V(x) P^{\prime}(\phi)
$$

with ' indicating partial derivation with respect to $\phi$.

The model can also be formulated on curved domains by replacing all operators by their surface counterparts.

\section{Numerical methods}

All computations were done using the finite element toolbox AMDiS. ${ }^{32}$ AMDiS allows for numerical solution of a large class of stationary and non-stationary systems of partial differential equations. It supports, among others, adaptivity in space and time, higher order and mixed finite elements as well as a framework for dimensionless problem specification. AMDiS fully supports the usage of high performance computer (HPC) systems and has been proven to scale efficiently on a large number of cores. ${ }^{33}$

The numerical experiments presented here make all use of adaptive grids. The initial configuration is interpolated on a fine grid. As coarsening of the solution proceeds in time the mesh coarsens as well. Our coarsening and refinement algorithm ensures that at least 15 mesh nodes lie inside the diffuse interface of width $\omega$. Away from the interface the mesh can become as coarse as possible. The modified Cahn-Hilliard equation is solved as a system of two second order equations, which are discretized in space using linear finite elements for each component $\phi$ and $\mu_{\mathrm{PF}}$ and in time using a Rosenbrock method of third order (ROWDA3), see ref. 34-36 for details.

The FETI-DP ${ }^{33,37}$ domain decomposition method is used to parallelize the problem. The computations were done on the HPC system JUROPA at the Jülich Supercomputing Center (JSC, Germany). As the mesh becomes coarser in time, we use an adaptive number of cores for the computations. All computations are started with 64 cores and the number of used cores is decreased during simulation to ensure that each subdomain contains at least 100000 mesh nodes, which ensure efficiency.

\section{Results}

\subsection{Domain coarsening}

We first consider the situation without pinnings. We are concerned with interfacial dynamics of the domain boundaries. When the domains are formed, the free energy of the system is concentrated on the interfaces as an effect of line tension. Since the system tries to minimize the energy, the amount of interfacial area decreases in time. This is realized by coarsening of the domain distribution. The characteristic length scale of the system, which describes the typical size of the domains, increases. In the interfacial dynamics of a two-component system two interesting features are numerically and experimentally observed: the morphology behaves statistically self-similar in time, and the growth rate of the characteristic length scale obeys a temporal power law. These investigations are confirmed by analytical results of the sharp interface limit of the considered phase-field approach.

The LSW approach ${ }^{38,39}$ treats an ensemble of coarsening domains and makes quantitative predictions on the long-time behaviour of coarsening systems. The morphology of the dispersed phase is thereby characterized in terms of a domain radius distribution $f(r, t)$, where $f$ is defined as the number of domains per unit volume at time $t$ in a size class $r$ to $r+\delta r$. The time rate of change of $f$ is therefore given by $\partial_{t}+\partial_{r}(f \dot{r})=0$. Under the assumption of an approximately constant chemical potential $\bar{u}$ far away from the clusters one obtains $\dot{r}=\bar{u} / r-1 / r^{2}$ and $\bar{u}=\int f \mathrm{~d} r / \int r f \mathrm{~d} r$. Asymptotic analysis predicts a universal long-time behaviour of the cluster radii distribution $\partial_{t} f$. The average radius $\bar{r}=1 / \bar{u}$ grows like $\bar{r}(t)=(4 t / 9)^{1 / 3}$ independently of the initial configuration. The derivation of the LSW theory assumes a non-existing long-range interaction of domains. This is only true in the unrealistic limit of the zero volume fraction $\xi$ of the coarsening phase. Extensions of the LSW theory to nonzero coverage $\xi$ are given i.e. in ref. 40 and 41 and the references therein. In ref. 42 it is analytically shown that the long-time behaviour in this case is not universal, but sensitively depends on the behaviour of the initial distribution. The modified growth rate in all these approaches is given by $\bar{r}(t)=(K(\xi) t)^{1 / 3}$ where the coarsening rate $K(\xi)$ is a monotonically increasing function of coverage $\xi$. The extension of the three dimensional LSW theory to two dimensions is due to the 
divergence of the logarithmic Green's function, which is less straightforward. Derivations rely on a cutoff at some ad hoc distance from the islands or on an introduced screening function. ${ }^{43} \mathrm{~A}$ rigorous derivation in the two-dimensional setting is given in ref. 44. If $d$ and $r$ are defined such that $\xi:=\pi(r / d)^{2}$ is the area fraction, for $\xi \ll 1$ one obtains highest order $\dot{r} \approx 1 / \ln \left(1 / \xi^{1 / 2}\right) \bar{u} / r-1 / r^{2}$ with $\bar{u}=\int f / r \mathrm{~d} r / \int f \mathrm{~d} r$. Thus the kinetic equation for $f$ reads $\ln \left(1 / \xi^{1 / 2}\right) \partial_{t} f+\partial_{r}(f \dot{r})=0$. Note that $\bar{u}$ can become singular. Again a scaling law of the form $\bar{r}(t)=(K(\xi) t)^{1 / 3}$ can be derived, with $K(\xi)$ diverging as $1 / \ln \left(\xi^{-1 / 2}\right)$ as $\xi \rightarrow 0$.

We first use these analytical results to validate our model. We prefer to work with a phase-field version of the underlying model, the Cahn-Hilliard equation. ${ }^{45}$ This has the advantage that the Cahn-Hilliard equation can be directly derived as a mean-field model for Kawasaki dynamics in the Ising model and therefore allows a comparison with previous studies. In the limit of vanishing interfacial thickness $\omega$ one obtains the sharp interface model the LSW-theory is based on. One can thus expect similar scaling behaviour also for the CahnHilliard equation. In ref. 46 the large time regime of the Cahn-Hilliard equation is analysed. Rigorous upper bounds for the coarsening rate in the Cahn-Hilliard equation are derived which demonstrate that the characteristic length $r$ cannot coarsen faster than $t^{1 / 3}$. Lower bounds for the coarsening rate have not been derived rigorously and can also not be expected within a deterministic framework, as there might exist configurations with zero curvature, for which coarsening will not occur. Computational studies which concentrate on the transient behaviour of the coarsening have e.g. been done in ref. 47 . We first confirm these results. Fig. 3 shows numerical simulations demonstrating the theoretically expected $t^{-1 / 3}$ scaling for the interface length. The derivation for large times results from the influence of the boundary.

As a second result Fig. 4 shows the island size distribution function obtained in our simulations in comparison with the LSW theory. The scaled island size distribution is time-independent within the coarsening regime. In agreement with the theoretical results for non-zero coverage and the simulation results in ref. 48 a broadening of the island size distribution function with increasing coverage is observed.

The observed scaling is in agreement with the belonging of phase separation in giant vesicles to the universality class of a two-dimensional Ising model ${ }^{49}$ for which $\bar{r}(t) \approx t^{1 / 3}$. In ref. 18 it is shown that in the presence of pinned obstacles the universality class changes and membranes with immobile proteins belong to the universality class of a two-dimensional random-field Ising model, which is significantly different as it does not allow for macroscopic phase separation and thus gives an elegant explanation for the occurrence of heterogeneous equilibrium domains. The universality class of a two-dimensional random-field Ising model was shown to be applicable for randomly distributed pinning of membrane proteins with a preferred affinity to one of the lipid phases. One prediction of the random-field Ising model is a significant slow down of the growth law $\bar{r}(t) \approx t^{1 / 3}$ at intermediate stages and a cross over to an extremely slow logarithmic growth $\bar{r}(t) \log t$ which leads to an equilibrium state in an experimental time, with the domain size determined by the pinning density and pinning strength.

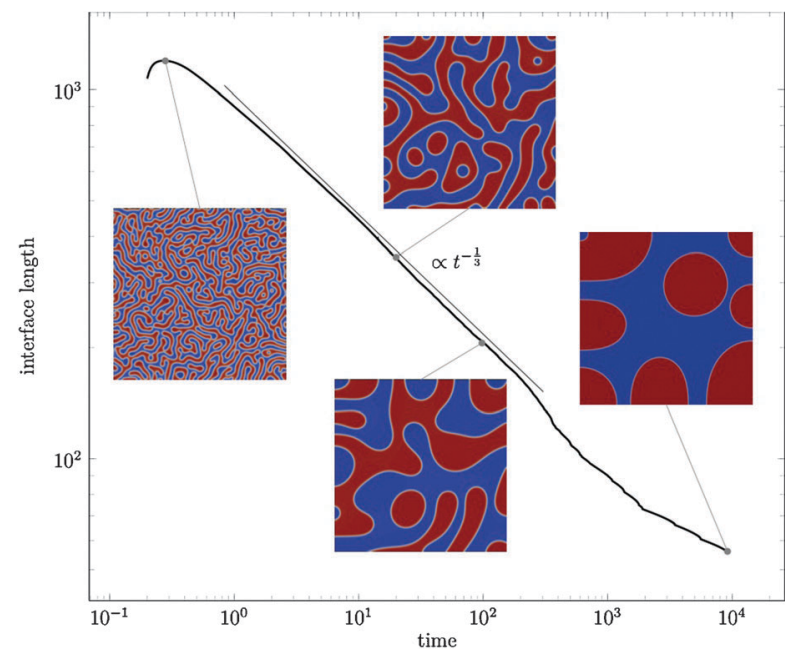

Fig. 3 Instead of the characteristic length $r$ we here measure the interface length of the domain boundary. An equal distribution of both phases is used which is randomly perturbed to form an initial condition. Zero-flux boundary conditions are used. There are three different stages: spinodal decomposition, followed by coarsening and finally reaching a stationary configuration. Other distributions of the two phases (40:60 and $30: 70$ ) lead to similar scaling results, but show an even more pronounced intermediate regime with a smaller coarsening exponent before the system settles into the $t^{-1 / 3}$ scaling.

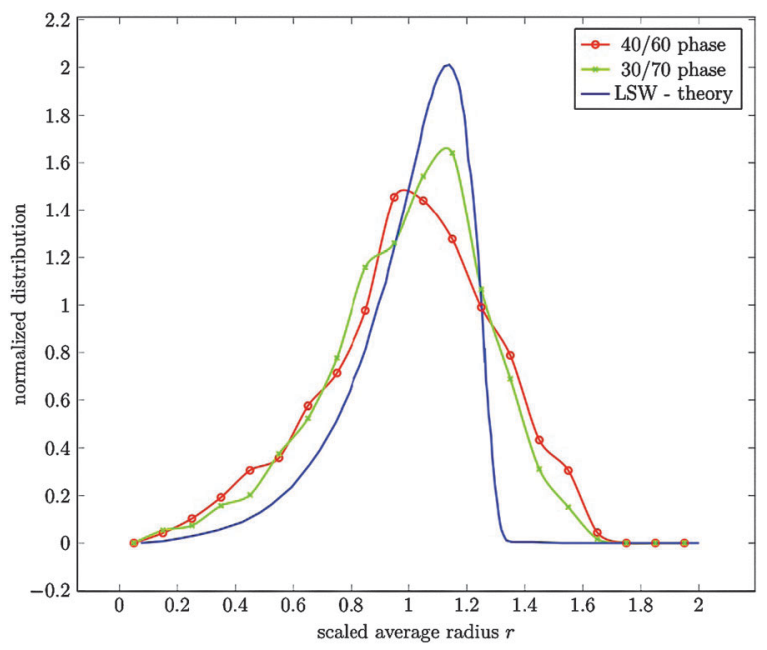

Fig. 4 We consider a distribution of $40: 60$ and $30: 70$ for the two phases. A homogeneous field is randomly perturbed to form an initial condition. Zero-flux boundary conditions are used. We compare the computed island size distribution function with that of the LSW theory. The size distribution is rescaled in each time-step with respect to the average size of an island at this time step. Shown is the average over all time steps within the considered time interval. Islands in contact with the domain boundary are not considered.

This could be reproduced in ref. 10. It is also in agreement with a significant slow-down of the coarsening in the presence of quenched impurities in binary mixtures, see e.g. ref. 50. However, the modeling approach to study domain growth with quenched disorder differs, as a random-bond Ising model is used.

A phase field analogon to the studies in ref. 10 and 18 is described above. Theoretical results for the modified CahnHilliard equation or its sharp interface limit are not known. 
We will investigate the behaviour numerically using large scale simulations. In the field of domain growth with quenched disorder, phase field simulations have been performed, see e.g. ref. 51. However, as for Monte Carlo methods the modeling approach differs, by perturbing the free energy globally instead of a localized pinning as in our case.

\subsection{Results with pinnings}

Also with pinnings the dynamics of the phase separation process is characterized by three states. (i) An initial state showing the spinodal decomposition after quenching, (ii) a coarsening state, which might involve a cross-over between different states characterized by different scaling exponents, and (iii) reaching an equilibrium state. We analyse them for an increasing number of pinning sites. Thereby the pinning sites are such that they prefer one lipid phase, they are randomly distributed and have the same size. With this configuration we fulfill the requirement of ref. 18 for the universality class of a two-dimensional random-field Ising model. We observe a slow down of the domain growth which increases with the number of pinning sites. Fig. 5 shows the corresponding scaling behaviour.

The scaling law differs from the evolution without pinnings. We observe a decrease in the coarsening process, which becomes slower and slower with increasing number of pinning sites. Even more pronounced is the difference in the final configuration, which shows smaller structures for increasing number of pinning sites. Also a turn-over point from the observed scaling to a drastically slowed down coarsening phase before the

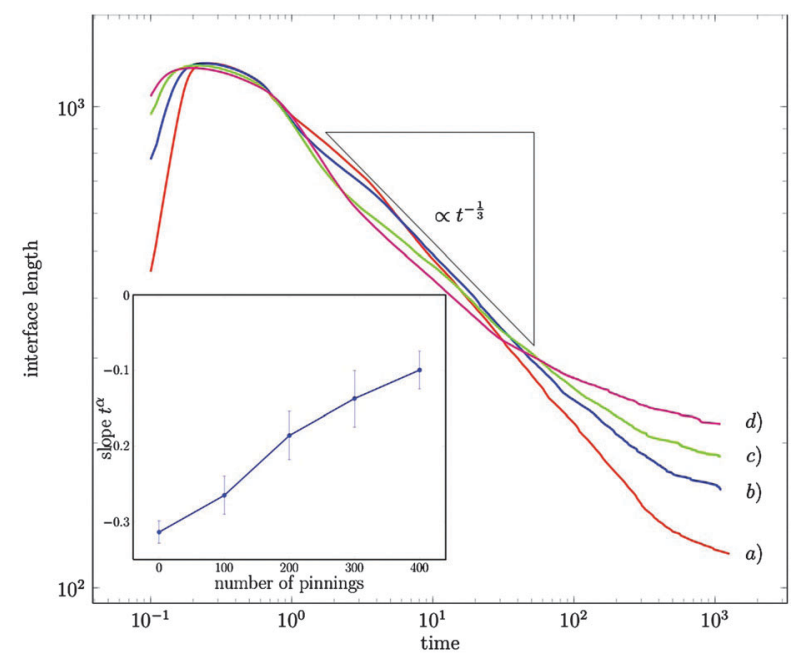

Fig. 5 The interface length of the domain boundary is considered as a function of pinning sites. An equal distribution of both phases is used which is randomly perturbed to form an initial condition. Zeroflux boundary conditions are used. The number of pinning sites varies between (a) 100, (b) 200, (c) 300 and (d) 400. They are randomly distributed. There are three different stages: spinodal decomposition, followed by coarsening and finally reaching a stationary configuration. The spinodal decomposition stage is almost identical for all configurations. The theoretical scaling exponent without pinning sites is shown for comparison. The inset shows the obtained scaling exponent as a function of pinning sites. The error bars result from the considered interval to compute the exponent. The interface length of the final configuration increases with the number of pinning sites.
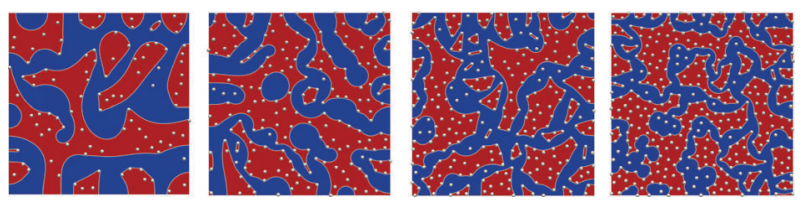

Fig. 6 Final configuration from Fig. 5 for (a) 100, (b) 200, (c) 300 and (d) 400 pinning sites, from left to right.

final configuration is reached, it differs for different pinning numbers and is reached earlier for higher numbers of pinning sites. The final configurations are shown in Fig. 6.

We obtain similar scaling results also for other phase distributions. As a final result we consider the island size distribution under the influence of pinnings. As predicted within the random field Ising model self-similarity should no longer be present, which indeed is the case in our simulations also. We show this in Fig. 7 by plotting the size distribution for various time steps. They significantly differ from the results without pinnings. In all cases a broadening with increasing time is observed. The large peak in the island size distribution resamples islands which form around a pinning site. The position of the peak is fixed and corresponds to the size of the pinning sites. The self-similarity cannot be achieved. We therefore do not rescale the size distribution. Instead we plot the obtained distribution for different times and compare them to each other and the corresponding distribution without pinning.

The results in each row shown in Fig. 7 are obtained from one simulation run, which explains the large deviations between the selected times in the case without pinnings. An average over various simulations with different initial conditions would smooth these differences. However, also for a single simulation a significant movement towards larger island sizes with increasing time can be clearly observed. This demonstrates the coarsening behaviour of the system and the development

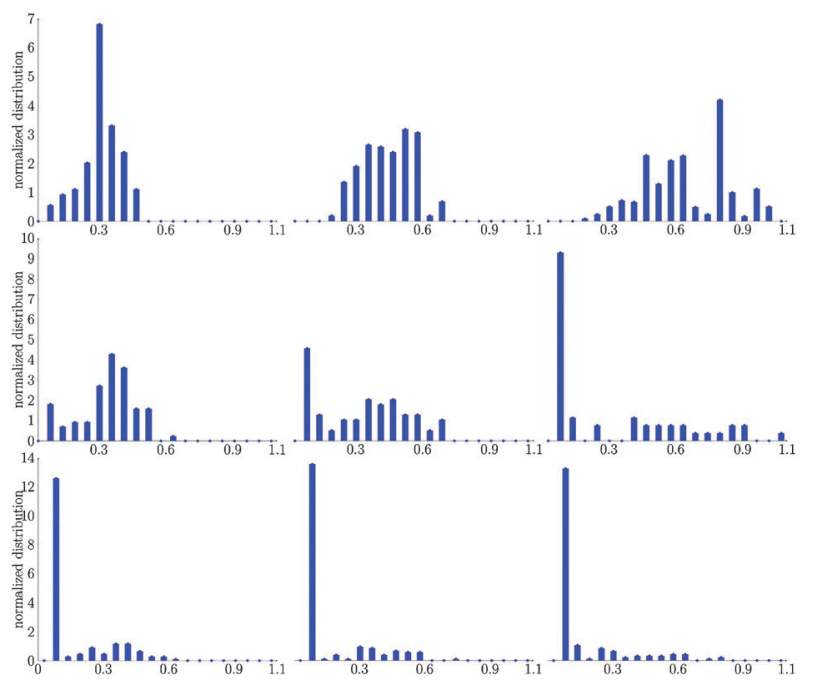

Fig. 7 Island size distribution, which is shown in comparison with the island size distribution function obtained without pinning sites. We do not rescale the island sizes as no self-similarity can be expected. (top row) Distribution without pinnings, (middle row) distribution with 100 pinnings and (bottom row) distribution with 400 pinnings for increasing time from left to right. 
towards macroscopically sized islands. With pinnings this is no longer the case. The island size distribution broadens in time and with increasing number of pinnings. In the presence of pinnings the distribution fills the whole range of island sizes, which demonstrates a breakdown of coarsening and the persistence of heterogeneity on small scales which can be interpreted as raft domains.

\section{Conclusions}

In ref. 10 and 18 it was shown that quenched obstacles within a membrane drastically change the coarsening behaviour. By relating domain formation in such systems to universality classes it was shown in ref. 18 that membranes without quenched obstacles belong to the universality class of a two dimensional Ising model, while the presence of obstacles changes the situation to the universality class of a two dimensional random-field Ising model. Macroscopic phase separation no longer occurs and the remaining phase consists of microdomains. This is in agreement with the heterogeneity in cell membranes on a micrometer length scale and provides an elegant explanation of the lipid raft hypothesis. Inspired from these findings we perform mean-field simulations for similar configurations. Corresponding to the two-dimensional Ising model a Cahn-Hilliard equation is considered for which large scale simulations reproduced the known coarsening properties of self-similarity and scaling. Modifying the Cahn-Hilliard model by introducing additional pinning sites resamples the main properties of the two-dimensional random-field Ising model. The pinning sites are introduced using an additional potential which locally prefers one of the two phases. Large scale simulations with randomly distributed pinning sites show a significant difference in the phase separation process when compared with the situation without pinnings. The coarsening process is significantly slowed down with an exponent depending on the density of pinning sites. Still domains dynamically change their shape, but they stay pinned to the pinning sites and eventually large-scale phase separation is prevented. The final domain size is largely determined by the density of the pinning sites. Our simulations predict that coarsening is strongly slowed down at intermediate stages and crosses over to an extremely slow logarithmic coarsening. Domain growth stops, when domains reach a characteristic size determined by the pinnings. Such behaviour is in agreement with the findings in ref. 10-12 and is also similar to results obtained in other systems, such as phase-separating polymer gels and binary alloys with impurities. ${ }^{51}$ In addition to the scaling behaviour we computed an island size distribution which is no longer self-similar and shows a strong broadening in time, with a distribution over the whole range of island sizes. This further confirms the breakdown of self-similarity and coarsening towards macroscopic domains.

The introduced pinning sites, which resample membrane bound proteins, clearly demonstrate a difference in the phase separation behaviour and allow us to explain the difference of experimental results in vitro and in vivo, especially large-scale phase separation and dynamically stable microdomains. Being able to observe this within a mean field model allows us to further incorporate additional effects, such as membrane curvature, line tension and flow and the efficient solution of the system on scales of relevance for further experimental studies. Furthermore it would be possible to consider the effect of proteins, with a preference for different lipid domains, proteins with different sizes as well as proteins which are able to move along the membrane.

We acknowledge financial support from DFG through grant Vo899/6, from EU through grant PHASEFIELD and computing resources at the high performance computer JUROPA through grant HDR06.

\section{References}

1 K. Simons and E. Ikonen, Nature, 1997, 387, 569-572.

2 H. T. McMahon and J. L. Gallop, Nature, 2005, 438, 590-596.

3 S. L. Veatch and S. L. Keller, Biophys. J., 2003, 85, 3074-3083.

4 S. L. Veatch, O. Soubias, S. L. Keller and K. Gawrisch, Proc. Natl. Acad. Sci. U. S. A., 2007, 104, 17650-17655.

5 A. Yethiraj and J. C. Weisshaar, Biophys. J., 2007, 93, 3113-3119.

6 J. Fan, M. Sammalkorpi and M. Haataja, FEBS Lett., 2010, 584, $1678-1684$.

7 J. Fan, M. Sammalkorpi and M. Haataja, Phys. Rev. Lett., 2010, 104, 118101.

8 R. Brewster and S. A. Safran, Biophys. J., 2010, 98, L21-L23.

9 W. Ngamsaad, S. May, J. Wagner and W. Triampo, Soft Matter, 2011, 7, 2848-2857.

10 J. Ehrig, E. P. Petrov and P. Schwille, Biophys. J., 2011, 100, 80-89.

11 J. Gómez, F. Sagués and R. Reigada, J. Chem. Phys., 2010, 132, 135104.

12 J. Ehrig, E. Petrov and P. Schwille, New J. Phys., 2011, 13, 045019.

13 Y. Imry and S. Ma, Phys. Rev. Lett., 1975, 35, 1399-1401.

14 M. Laradji and P. B. S. Kumar, J. Chem. Phys., 2005, 123, 224902.

15 J. Fan, T. Han and M. Haataja, J. Chem. Phys., 2010, 133, 235101.

16 B. Camley and F. Brown, J. Chem. Phys., 2011, 135, 225106.

17 A. Kusumi, K. G. N. Suzuki, R. S. Kasai, K. Ritchie and T. K. Fujiwara, Trends Biochem. Sci., 2011, 36, 604-615.

18 T. Fischer and R. Vink, J. Chem. Phys., 2011, 34, 055106.

19 A. Rätz and A. Voigt, Commun. Math. Sci., 2006, 4, 575-590.

20 C. Varea, J. Aragon and R. Barrio, Phys. Rev. E: Stat. Phys., Plasmas, Fluids, Relat. Interdiscip. Top., 1999, 60, 4588-4602.

21 P. Tang, F. Qiu, H. Zhang and Y. Yang, Phys. Rev. E: Stat. Phys., Plasmas, Fluids, Relat. Interdiscip. Top., 2005, 72, 016710.

22 M. Bowick and L. Giomi, Adv. Phys., 2009, 58, 449-563.

23 R. Backofen, A. Voigt and T. Witkowski, Phys. Rev. E: Stat. Phys., Plasmas, Fluids, Relat. Interdiscip. Top., 2010, 81, 025701.

24 X. Wang and Q. Du, J. Math. Biol., 2008, 56, 347-371.

25 J. Lowengrub, A. Rätz and A. Voigt, Phys. Rev. E: Stat. Phys., Plasmas, Fluids, Relat. Interdiscip. Top., 2009, 79, 031926.

26 T. Baumgart, S. Hess and W. Webb, Nature, 2003, 425, 821-824.

27 I. Nitschke, A. Voigt and J. Wensch, J. Fluid Mech, accepted for publication.

28 C. M. Elliott and B. Stinner, J. Comput. Phys., 2010, 229, 6585-6612.

29 C. M. Elliott and B. Stinner, SIAM J. Appl. Math., 2010, 70, 2904-2928.

30 M. Laradji, H. Guo, M. Grant and M. Zuckermann, J. Phys.: Condens. Matter, 1992, 4, 6715-6728.

31 J. Fan, M. Sammalkorpi and M. Haataja, Phys. Rev. E: Stat. Phys., Plasmas, Fluids, Relat. Interdiscip. Top., 2010, 81, 011908.

32 S. Vey and A. Voigt, Comput. Visualization Sci., 2007, 10, 57-67.

33 A. Voigt and T. Witkowski, J. Comput. Sci., http://dx.doi.org/ 10.1016/j.jocs.2012.06.004.

34 M. Roche, Numerische Mathematik, 1987, 52, 45-63.

35 S. Hartmann and J. Wensch, Comput. Mech., 2007, 40, 383-398.

36 B. Li, J. Lowengrub, A. Rätz and A. Voigt, Commun. Comput. Phys., 2009, 6, 433-482.

37 A. Klawonn and O. Rheinbach, Comput. Methods Appl. Mech. Eng., 2007, 196, 1400-1414.

38 L. Lifshitz and V. Slyozov, J. Phys. Chem. Solids, 1961, 19, 35-50.

39 A. Wagner, Z. Elektrochem., 1961, 65, 581-591.

40 P. Voorhees, J. Stat. Phys., 1985, 38, 231-252.

41 A. Ardell, Phys. Rev. B: Condens. Matter Mater. Phys., 1990, 41, 2554-2556. 
42 B. Niethammer and R. Pego, J Stat. Phys., 1999, 95, 867-902.

43 J. Marqusee, J. Chem. Phys., 1984, 81, 976-989.

44 B. Niethammer and F. Otto, Calculus Var. Partial Differ. Equations, 2001, 68, 33-68.

45 J. W. Cahn and J. E. Hilliard, J. Chem. Phys., 1958, 28, 258-267.

46 R. V. Kohn and F. Otto, Commun. Math. Phys., 2002, 395, 375-395.

47 H. Garcke, B. Niethammer, M. Rumpf and U. Weikard, Acta Mater., 2003, 51, 2823-2830.
48 F. Haußer and A. Voigt, Phys. Rev. B: Condens. Matter Mater. Phys., 2005, 72, 035437.

49 A. Honerkamp-Smith, S. Veatch and S. Keller, Biophys. J., 2009, 1788, 53-63.

50 D. Huse and C. Henley, Phys. Rev. Lett., 1985, 25, 2708-2711.

51 S. C. Glotzer, M. F. Gyure, F. Sciortino, A. Coniglio and H. E. Stanley, Phys. Rev. E: Stat. Phys., Plasmas, Fluids, Relat. Interdiscip. Top., 1994, 49, 247-258. 